\title{
FATORES BIOPSICOSSOCIAIS EM IDOSOS, DE ALTA VULNERABILIDADE SOCIAL, COM DOR LOMBAR CRÔNICA INESPECÍFICA
}

Maria Júlia da Cruz Souza, Universidade Federal de São Carlos, mariasouza@estudante.ufscar.br José Salvador Ribeiro Marques, Universidade Federal de São Carlos, salvinha123@gmail.com Letícia Souza Didoné, Universidade Federal de São Carlos, leticiadidone1@gmail.com Isabela Thais Machado de Jesus, Universidade Federal de São Carlos, isabela.machado1@gmail.com Marisa Silvana Zazzetta, Universidade Federal de São Carlos, marisam@ufscar.br Fabiana de Souza Orlandi, Universidade Federal de São Carlos, forlandi@ufscar.br Karina Gramani-Say, Universidade Federal de São Carlos, gramanisay@ufscar.br

\section{RESUM0}

Introdução: A dor lombar crônica inespecífica (DLCI) é a principal causa mundial de anos perdidos por incapacidade. A DLCI pode estar relacionada a aspectos biopsicossociais exacerbados com a vulnerabilidade social e que prejudicam a capacidade funcional. O conhecimento desses fatores auxilia na elaboração do plano de cuidado da DCLI. Objetivo: Avaliar os fatores biopsicossociais em idosos com DLCI em vulnerabilidade social. Métodos: Trata-se de um estudo transversal no qual participaram 158 idosos, divididos em dois grupos: Grupo Com Dor Lombar (GCDL) $(\mathrm{n}=104)$ e Grupo Sem Dor Lombar (GSDL) $(\mathrm{n}=52)$. Foi utilizado o banco do Programa Pesquisa Para o SUS (PPSUS), os idosos residiam em uma área de alta vulnerabilidade social, apresentavam compreensão para responder as perguntas e relato de dor na região lombar há mais de seis meses (CEP: 3.291.232/2019). As variáveis avaliadas foram Intensidade da Dor, Locais de Dor, Atividades de Vida diária, Mobilidade e Sintomas Depressivos. Foi realizado o Teste de Mann-Whitney ( $\mathrm{p} \leq 0,05)$ (SPSS Statistics 22.0). Resultados: Houve diferença significativa no Teste Timed Up and Go (TUG), sendo que o GCDL apresentou pior desempenho $(12,32 \pm 4,09 \mathrm{~s})$ em relação ao GSDL $(11,35 \pm 3,39 \mathrm{~s})$. O GCDL também apresentou diferença por não realizar 30 minutos de atividade física diária. Conclusão: Os idosos com DLCI apresentam pior desempenho em relação à mobilidade e equilíbrio, estando mais propensos ao risco de quedas e não realizam atividade física regularmente. Pode-se considerar que a presença de dor crônica é influenciada pela inatividade física e a DLCI tem influência negativa na mobilidade em idosos em alta vulnerabilidade social.

Palavras-chave: Envelhecimento; Dor Lombar; Dor Crônica.

Agradecimentos: Agradecimentos pelo financiamento da pesquisa ao Conselho Nacional de Desenvolvimento Científico e Tecnológico (CNPq), a Fundação de Amparo a Pesquisa do Estado de São Paulo (FAPESP) e a Coordenação de Aperfeiçoamento de Pessoal de Nível Superior (CAPES). 